\title{
Some Fixed Point Theorems for Fuzzy Iterated Contraction Maps in Fuzzy Metric Spaces
}

\author{
Lei Xia, Yuehan Tang \\ College of Mathematics, Physics and Information Engineering, Jiaxing University, Jiaxing, China \\ Email:Leixia0001@126.com, TangJohn@126.com
}

How to cite this paper: Xia, L. and Tang, Y.H. (2018) Some Fixed Point Theorems for Fuzzy Iterated Contraction Maps in Fuzzy Metric Spaces. Journal of Applied Mathematics and Physics, 6, 224-227. https://doi.org/10.4236/jamp.2018.61021

Received: December 28, 2017

Accepted: January 26, 2018

Published: January 29, 2018

Copyright $\odot 2018$ by authors and Scientific Research Publishing Inc. This work is licensed under the Creative Commons Attribution International License (CC BY 4.0).

http://creativecommons.org/licenses/by/4.0/

\begin{abstract}
The purpose of this paper is to introduce the notion of fuzzy iterated contraction maps in fuzzy metric spaces and establish some fixed point theorems for fuzzy iterated contraction maps in fuzzy metric spaces.
\end{abstract}

\section{Keywords}

Fixed Point, Iterated Contraction Map, Fuzzy Metric Space

\section{Introduction}

In 1975, Kramosil and Michalek [1] first introduced the concept of a fuzzy me(c) (1) Open Access tric space. In 1994, George and Veeramani [2] slightly modified the concept of fuzzy metric space introduced by Kramosil and Michalek, defined a Hausdorff topology and proved some known results. In 1969, Rheinboldt [3] initiated the study of iterated contraction. The concept of iterated contraction proves to be very useful in the study of certain iterative process and has wide applicability in metric spaces. In this paper we introduce the notion of fuzzy iterated contraction maps in fuzzy metric spaces and establish some fixed point theorems for fuzzy iterated contraction maps in fuzzy metric spaces.

\section{Preliminaries}

Definition $2.1([2])$. A fuzzy metric space is an ordered triple $(X, M, *)$ such that $X$ is a (nonempty) set, $*$ is a continuous $t$-norm and $M$ is a fuzzy set on $X \times X \times(0, \infty)$ satisfying the following conditions, for all $x, y, z \in X, \quad s, t>0$ :

(FM-1) $M(x, y, t)>0$;

(FM-2) $\quad M(x, y, t)=1$ if and only if $x=y$;

(FM-3) $M(x, y, t)=M(y, x, t)$;

(FM-4) $M(x, y, t) * M(x, z, t+s)$; 
$(\mathrm{FM}-5) \quad M(x, y, \bullet):(0, \infty) \rightarrow(0,1)$ is continuous.

Definition 2.2 ([2]). A map $T: X \rightarrow X$, satisfying $M(T x, T y, t) \geq M\left(x, y, \frac{t}{k}\right)$, for all $x, y \in X, t>0,0<k<1$, is called a contraction map.

\section{Main Results}

In this part, we firstly give a notion of the fuzzy iterated contraction map in a fuzzy metric space, then we prove some fixed point theorems for fuzzy iterated maps under different settings.

Definition 3.1 If $(X, M, *)$ is a fuzzy metric space such that $M\left(T x, T^{2} x, t\right) \geq M\left(x, T x, \frac{t}{k}\right)$ for all $x \in X, t>0,0<k<1$, then $T$ is said to be a fuzzy iterated contraction map.

Remark 3.1 A fuzzy contraction map is continuous and is a fuzzy iterated contraction. A fuzzy contraction map has a unique fixed point. However, a fuzzy iterated contraction map may have more than one fixed point.

Let $(X, d)$ be a metric space, Define $x * y=x y$ and $d(x, y)=|x-y|$ for all $x, y \in X$ and $t>0$,

$$
M(x, y, t)=\frac{t}{t+d(x, y)}
$$

then $(X, M, *)$ is a fuzzy metric space.

If $T:[-1 / 2,1 / 2] \rightarrow[-1 / 2,1 / 2]$ is given by $T x=x^{2}$, then $T$ is a fuzzy iterated contraction but not a fuzzy contraction map.

The following is a fixed point theorem for fuzzy iterated contraction map.

Theorem 3.1 If $T: X \rightarrow X$ is a continuous fuzzy iterated contractive map and the sequence of iterates $\left\{x_{n}\right\}$ in fuzzy metric space $(X, M, *)$, defined by $x_{n+1}=T x_{n}, n=1,2, \cdots$ for $x \in X$, has a subsequence converging to $y \in X$, then $y=T y$, that is, $T$ has a fixed point.

Proof: The sequence $\left\{M\left(x_{n+1}, x_{n}, t\right)\right\}$ is a nondecreasing sequence of reals. It is bounded above by 1 , and therefore has a limit. Since the subsequence converges to $y$ and $T$ is continuous on $X$, so $T\left(x_{n_{i}}\right)$ converges to $T y$ and $T^{2}\left(x_{n_{i}}\right)$ converges to $T^{2} y$.

Thus $M(y, T y, t)=\lim M\left(x_{n_{i}}, x_{n_{i+1}}, t\right)=\lim M\left(x_{n_{i+1}}, x_{n_{i+2}}, t\right)=M\left(T y, T^{2} y, t\right)$ then

$$
\begin{aligned}
1 & \geq M\left(T y, T^{2} y, t\right) \geq M\left(y, T y, \frac{t}{k}\right) \\
& \geq M\left(y, T y, \frac{t}{k^{2}}\right) \geq \cdots \\
& \geq M\left(y, T y, \frac{t}{k^{n}}\right), \forall n \in N, 0<k<1
\end{aligned}
$$

when $n \rightarrow \infty, M\left(y, T y, \frac{t}{k^{n}}\right) \rightarrow 1$, therefore $M\left(y, T y, \frac{t}{k}\right)=1$ that is to say 
$T y=y$.

We give the following example to show that if $T$ is a fuzzy iterated contraction that is not continuous, then $T$ may not have a fixed point.

A continuous map $T$ that is not a fuzzy iterated contraction may not have a fixed point.

Note 3.1 If $T$ is not contraction but some powers of $T$ is contraction, then $T$ has a unique fixed point on a complete metric space.

Proof: If $x$ is a fixed point of $k$ powers of $T$, thus $T^{k}(x)=x$, $T\left(T^{k}(x)\right)=T(x)$, then $T^{k}(T(x))=T(x)$, since $T^{k}$ has a unique fixed point. Consequently $T(x)=x$.

Note 3.2 Continuity of a fuzzy iterated contraction is sufficient but not necessary.

As stated in Note 3.1 that if $T$ is not contraction still $T$ may have a unique fixed point when some powers of $T$ is a fuzzy contraction map. The same is true for fuzzy iterated contraction map.

Theorem 3.2 Let $T: X \rightarrow X$ be a fuzzy iterated contraction map on a complete metric space $X$. If for some power of $T$, say $T^{r}$ is a fuzzy iterated contraction, that is, $M\left(T^{r} x,\left(T^{r}\right)^{2} x, t\right) \geq M\left(x, T^{r} x, \frac{t}{k}\right)$ and $T^{r}$ is continuous at $y$, where $y=\lim \left(T^{r}\right)^{n} x$, for any arbitrary $x \in X$. Then $T$ has a fixed point.

Proof: Since $T$ is a fuzzy iterated contraction that is continuous at $y$,

$$
\begin{aligned}
& M\left(T^{r} x,\left(T^{r}\right)^{2}, t\right) \geq M\left(x, T^{r} x, \frac{t}{k}\right), 0<k<1 \\
M & \left(\left(T^{r}\right)^{n} x,\left(T^{r}\right)^{m} x, t\right) \\
\geq & \prod_{i=m}^{n} M\left(\left(T^{r}\right)^{i+1} x,\left(T^{r}\right)^{i} x, \frac{t}{n-m}\right), \forall n>m, n, m \in N \\
\geq & \prod_{i=m}^{n} M\left(x, T^{r} x, \frac{t}{(n-m) * k^{i-1}}\right) \rightarrow 1(m \rightarrow \infty)
\end{aligned}
$$

therefore $\left\{\left(T^{r}\right)^{n}\right\}_{n=1}^{\infty}$ is a Cauchy sequence in $X$ and $X$ is a complete metric space. Thus $\exists y \in X$, s.t. $y=\lim _{n \rightarrow \infty}\left(T^{r}\right)^{n} x$, and $T^{r}$ is continuous, consequently $T^{r}(y)=\lim _{n \rightarrow \infty} T^{r}\left(\left(T^{r}\right)^{n} x\right)=\lim _{n \rightarrow \infty}\left(T^{r}\right)^{n+1} x=y$.

It is easy to show that $d(y, f y) \leq k^{r} d(y, f y)$. Since $k^{r} \leq 1$, therefore $d(y, f y)=0$ and hence $f$ has a fixed point.

We give the following example to illustrate the theorem.

Note 3.3 If $T$ is not a fuzzy iterated contraction in Theorem 3.2, but $T^{r}$ is a fuzzy iterated contraction with $T^{r} y=y$, then $T$ has a fixed point.

Theorem 3.3 If $T: X \rightarrow X$ is a fuzzy iterated contraction map, and $X$ is a complete metric space, then the sequence of iterates $x_{n}$ converges to $y \in X$. In case, $T$ is continuous at $y$, then $y=T y$, that is, $T$ has a fixed point.

Proof: Let $x_{n+1}=T x_{n}, n=1,2, \cdots ; x_{1} \in X$. It is easy to show that $\left\{x_{n}\right\}$ is a 
Cauchy sequence, since $T$ is a fuzzy iterated contraction. The Cauchy sequence $\left\{x_{n}\right\}$ converges to $y \in X$, since $X$ is a complete metric space. Moreover, if $T$ is continuous at $y$, then $x_{n+1}=T x_{n}$ converges to Ty. It follows that $y=T y$.

Note 3.3 A continuous iterated contraction map on a complete metric space has a unique fixed point. If an iterated contraction map is not continuous, it may have more than one fixed point.

\section{Acknowledgements}

This paper is supported by the Student Research Training Program of Jiaxing University (No.851715034), the College Student's Science and Technology Innovation Project of Zhejiang Province (No.2016R417014).

\section{References}

[1] Kramosil, I. and Michálek, J. (1975) Fuzzymetric and Statistical Mestric Spaces. Kybernetika, 11, 336-334.

[2] George, A. and Veeramani, P. (1994) On Some Results in Fuzzy Metric Spaces. Fuzzy Sets and System, 64, 395-399. https://doi.org/10.1016/0165-0114(94)90162-7

[3] Rheinboldt, W.C. (1968) A Unified Convergence Theory for a Class of Iterative Process. SIAM Journal on Numerical Analysis, 5, 42-63.

https://doi.org/10.1137/0705003 